\title{
SOLUTIONS OF EQUATIONS OVER GROUPS
}

\author{
BY FRANK LEVIN
}

Communicated by Roger Lyndon, May 25, 1962

A polynomial equation of degree $n$ over a field $K$ can always be solved in a suitable extension $K^{\prime}$ of degree at most $n$ over $K$. One might expect to find a corresponding result for groups; it is the purpose of this note to show that this is, in fact, the case.

The analogous problem for groups is to find a solution for the equation

$$
x^{n_{1}} g_{1} x^{n_{2}} g_{2} \cdots x^{n k} g_{k}=1
$$

in the unknown $x$, where the $g_{i}$ are elements of a group $G$ and all the $n_{i}$ are non-negative, i.e., to find an element $g^{\prime}$ in a group $G^{\prime}$ in which $G$ is embedded such that

$$
g^{\prime n_{1}} g_{1} g^{\prime n_{2}} g_{2} \cdots g^{\prime n k} g_{k}=1
$$

in $G^{\prime}$. It will be shown that (1) can always be solved over any group $G$, with a solution in $G^{\prime}$, where $G^{\prime}$ is, in some sense, an extension group of "degree $n$ " over $G$, for $n=\sum_{i=1}^{k} n_{i}$. This result (coupled with the fact that the equation

$$
x^{-1} g_{1} x g_{2}=1
$$

is solvable over any torsion-free group [2]) gives hope for the conjecture that any equation is solvable over a torsion-free group.

The solution we will give for (1) uses the construction used by Baumslag [1] to solve the equation $x^{n} g=1$ (cf. [3]).

Without loss of generality, we may restrict our attention to equations of the form

$$
x a_{0} x a_{1} \cdots x a_{n-1}=1, \quad a_{i} \in G,
$$

where some of the $a_{i}$ are possibly 1 . The solution of (2) will be constructed in the wreath product of $G$ and a cyclic group $C$ of order $n$. To fix notation, we will outline the definition of the wreath product, GWrC (cf. [2]): Let $G^{c}$ be the group of all mappings $\{f\}$ of $C$ into $G$ with $f f^{\prime} \in G^{C}$ defined by $f f^{\prime}(t)=f(t) f^{\prime}(t)$, for all $t \in C$. GWrC is the group composed of the set $\left\{s f \mid s \in C, f \in G^{C}\right\}$ with

$$
s f \cdot s^{\prime} f^{\prime}=s s^{\prime} f^{\prime} f^{\prime},
$$

where $f^{\prime \prime}(t)=f\left(t s^{\prime-1}\right)$ for all $t \in C$. We will embed $G$ in $\mathrm{GWrC}$ by identifying $1^{\prime} g^{\circ} \in \mathrm{GWrC}$ with $g \in G$, where $g^{\circ}(t)=g$ for all $t \in C$ and $1^{\prime}$ is the neutral element of $C$. 
The main result of this note is stated as follows:

Theorem 1. Let $G$ be an arbitrary group, $C=g p(c)$ be a cyclic group of order $n$. A solution of equation (2) is given by $c^{-1} f \in \mathrm{GWrC}$, where $f\left(c^{i}\right)=a_{i}^{-1}, i=0,1, \cdots, n-1$.

In other words, Theorem 1 states that

$$
\left(c^{-1} f\right) \cdot\left(1^{\prime} a_{0}^{0}\right) \cdot\left(c^{-1} f\right) \cdot\left(1^{\prime} a_{1}^{0}\right) \cdots\left(c^{-1} f\right) \cdot\left(1^{\prime} a_{n-1}^{0}\right)=1^{\prime} 1^{0},
$$

( 1 is the neutral element of $G$ ), which can be verified by a straightforward application of the definition of $\mathrm{GWrC}$.

There are several properties of a group $G$ which are inherited by GWrC. For instance: if $G$ is finite, $\mathrm{GWrC}$ is finite; if $G$ has finite exponent $m, \mathrm{GWrC}$ has exponent $m n$; if $G$ is soluble, $\mathrm{GWrC}$ is soluble of length at most one greater than $G$.

A group which has the property that every equation of type (2) has a solution in the group itself will be called a $P$-algebraically closed group. Such a group is also divisible, i.e., contains a solution for every equation $x^{n} g=1$. Some of the results in [1] can be extended immediately to $P$-algebraically closed groups. In particular, Corollary 4.3 and Theorem 4.4 of [1], respectively, have the following extensions:

Corollary 1 (cf., also, Neumann [3]). Every group G can be embedded in a P-algebraically closed group.

Theorem 2. Every periodic group can be embedded in a periodic Palgebraically closed group.

For the solution of (2) it is sufficient, of course, to consider the subgroup $W^{\prime}$ of GWrC generated by $G$ and $c^{-1} f$ rather than the whole of GWrC. $W^{\prime}$ has, in fact, the same solubility length as $G$; this result can be checked rather easily here. Consequently, we can state

THEOREM 3. If $G$ is soluble of length $q$, a solution of equation (2) can be found in an overgroup $W^{\prime}$ of $G$ which is soluble of length $q$.

\section{REFERENCES}

1. G. Baumslag, Wreath products and p-groups, Proc. Cambridge Philos. Soc. 55 (1959), 224-231.

2. G. Higman, B. H. Neumann and Hanna Neumann, Embedding theorems for groups, J. London Math. Soc. 24 (1949), 247-254.

3. B. H. Neumann, Adjunction of elements to groups, J. London Math. Soc. 18 (1943), 12-20.

Rutgers, The State University 